\title{
DIVERSIDAD DE LAS GRAMÍNEAS (POACEAE) DE LIRCAY (ANGARAES, HUANCAVELICA, PERÚ)
}

\section{GRASSES (POACEAE) DIVERSITY OF LIRCAY (ANGARAES, HUANCAVELICA, PERU)}

\author{
Harol Gutiérrez Peralta ${ }^{1}$ y Roxana Castañeda Sifuentes ${ }^{2}$
}

\section{Resumen}

Para el distrito de Lircay se reporta un total de 46 especies y una subespecie de la familia Poaceae agrupadas en 21géneros, 11 tribus y 6 subfamilias. El género Calamagrostis es el más diverso con 9 especies, seguido por Poa con 5 especies. Asimismo, Aciachne acicularis "paccupaccu", Arundo donax "carrizo", Cortaderia hieronymi y Ortachne erectifolia "iruichu" constituyen nuevos registros para la región Huancavelica. Además, se presentan claves dicotómicas para la determinación de las especies en los casos de un género con más de una especie. Para cada especie se incluyen datos sobre su hábitat, distribución, material estudiado y nombre común.

Palabras clave: Aciachne, Arundo, Calamagrostis, Cortaderia, Orthacne, Poa, taxonomía.

\section{Abstract}

For the District of Lircay, we report a total of 46 species and one subspecies of the Poaceae family, grouped into 21genera, 11 tribes and 6 subfamilies. The genus Calamagrostis is the most diverse with 9 species, followed by Poa with 5 species. Also, Aciachne acicularis "paccupaccu", Arundo donax "carrizo", Cortaderia hieronymi and Ortachne erectifolia "iruichu" are new reports for the region of Huancavelica. Moreover, dichotomous keys are presented for the determination of species in the case a genus has more than one species. For each species, data on habitat, distribution, examined material and common name are included.

Key words: Aciachne, Arundo, Calamagrostis, Cortaderia, Orthacne, Poa, taxonomy.

\section{Introducción.}

La familia Poaceae está ampliamente distribuida en el mundo; sus especies se encuentran presentes en todas las latitudes y altitudes, desde el nivel del mar hasta por encima de los $5000 \mathrm{~m}$ (Tovar, 1993). Poaceae es una de las familias con mayor número de especies, con aproximadamente $\quad 700$ géneros y 10000 especies distribuidas en casi todos los continentes (Clayton \& Renvoize, 1986). En la flora peruana, está representada por alrededor de 157 géneros con 750 especies (Brako \& Zarucchi, 1993; Ulloa et al., 2004), que se encuentran ocupando todos los pisos bioclimáticos, desde las orillas del Océano
Pacífico hasta las altas cumbres de los Andes y desde éstas hasta la llanura amazónica atravesando los Andes orientales.

Si bien se han realizado estudios sobre las Poaceae

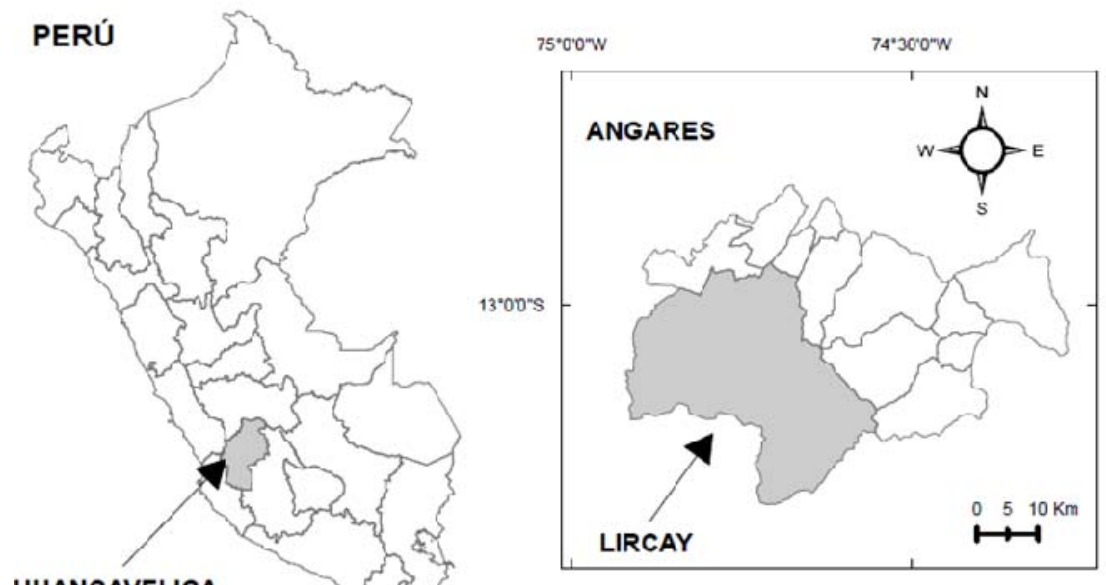

Figura 1. Mapa de ubicación del distrito de Lircay (Angaraes, Huancavelica, Perú). 
en la región, como los trabajos de Tovar (1957, 1960, 1965, 1972) en las provincias de Huancavelica y Tayacaja y registros de la provincia Castrovirreyna, no se cuenta con información sobre la diversidad agrostológica del distrito de Lircay (provincia de Angaraes). Por esta razón el objetivo del trabajo fue realizar un estudio sobre la diversidad específica de la familia Poaceae del distrito de Lircay con la finalidad de contribuir al conocimiento de la flora agrostológica huancavelicana e incrementar los números de registros y rangos de distribución de las especies de gramíneas para el Perú.

Materiales y métodos.

Área de Estudio

La zona de estudio comprende el distrito de Lircay en la provincia de Angaraes, región de Huancavelica (Figura 1), se encuentra en las coordenadas geográficas $12^{\circ}$ $59^{\prime} 23^{\prime}$ 'S y $74^{\circ} 43^{\prime} 14^{\prime}$ ' W, posee una extensión territorial de $1959.03 \mathrm{~km}^{2}$, en su geografía se puede observar pisos altitudinales que varían desde los $3000 \mathrm{~m}$ hasta los $4300 \mathrm{~m}$ (INEI, 2010).

La capital de Angaraes (Lircay) se encuentra situada en el extremo este de Huancavelica, sobre la margen derecha de la cuenca del río Lircay formada por sus afluentes, el Opamayo y Sicra, que discurren a través de la capital de distrito. Posee un clima típico de la sierra peruana, caracterizado por la alternancia estacional siendo: i) Época Seca (abril a noviembre), el clima frío se caracteriza por ser seco en invierno, con una temperatura media superior a $10^{\circ} \mathrm{C}$; ii) Época Húmeda (diciembre a marzo), el clima templado moderado lluvioso manifiesta un verano seco templado de
Tabla 1. Subfamilias, tribus y especies de Poaceae de Lircay (HuancavelicaPerú).

\begin{tabular}{|c|c|c|}
\hline Subfamilia & Tribu & Especie \\
\hline Aristidoideae & Aristideae & Aristida adscensionis L. \\
\hline Arundinoideae & Arundineae & Arundo donax L. \\
\hline \multirow[t]{7}{*}{ Chloridoideae } & Eragrostideae & Eragrostis sp. \\
\hline & & Eragrostis nigricans (Kunth) Steud. \\
\hline & Chlorideae & Bouteloua simplex Lag. \\
\hline & & Muhlenbergia fastigiata (J. Presl) Henrard \\
\hline & & Muhlenbergia ligularis (Hack.) Hitchc. \\
\hline & & Muhlenbergia peruviana (P. Beauv.) Steud \\
\hline & Zoysieae & Sporobolus indicus (L.) R. Br. \\
\hline Danthoniodeae & Danthonieae & $\begin{array}{l}\text { Cortaderia hieronymi N.P. Barker \& H.P. Linder } \\
\text { Cortaderia jubata (Lemoine) Stapf }\end{array}$ \\
\hline \multirow[t]{3}{*}{ Panicoidae } & Paniceae & $\begin{array}{l}\text { Cenchrus clandestinus (Hochst. ex Chiov.) Morrone. } \\
\text { Cenchrus weberbaueri (Mez) Morrone }\end{array}$ \\
\hline & Paspaleae & Paspalum pilgerianum Chase \\
\hline & & Paspalum tuberosum Mez \\
\hline \multirow[t]{32}{*}{ Pooideae } & Bromeae & Bromus catharticusVahl \\
\hline & & Bromus pitensis Kunth \\
\hline & Poeae & Avena sterilis L. \\
\hline & & Calamagrostis chrysantha (J. Presl) Steud. \\
\hline & & Calamagrostis intermedia (J. Presl) Steud. \\
\hline & & Calamagrostis jamesonii Steud. \\
\hline & & Calamagrostis minima (Pilg.) Tovar \\
\hline & & Calamagrostis ovata (J. Presl) Steud. \\
\hline & & Calamagrostis recta (Kunth) Trin. ex Steud. \\
\hline & & Calamagrostis rigescens (J. Presl) Scribn. \\
\hline & & Calamagrostis rigida (Kunth) Trin. ex Steud. \\
\hline & & Calamagrostis vicunarum (Wedd.) Pilg. \\
\hline & & Dactylis glomerata $\mathrm{L}$. \\
\hline & & Festuca dolichophylla J. Presl \\
\hline & & Festuca weberbaueri Pilg. \\
\hline & & Poa annua L. \\
\hline & & Poa calycina (J. Presl) Kunth \\
\hline & & Poa horridula Pilg. \\
\hline & & Poa lepidula (Nees \& Meyen) Soreng \& L.J. Gillespie \\
\hline & & Poa serpaiana Refulio \\
\hline & & Polypogon elongatus Kunth \\
\hline & & Polypogon interruptus Kunth \\
\hline & & Vulpia australis (Nees ex Steud.) C.H. Blom \\
\hline & & Vulpia myuros (L.) C.C. Gmel. \\
\hline & & Vulpia myuros var. hirsuta Hack. \\
\hline & Stipeae & Aciachne acicularis Lægaard \\
\hline & & Aciachne pulvinata Benth. \\
\hline & & Jarava ichu Ruiz \& Pav. \\
\hline & & Nassella meyeniana (Trin. \& Rupr.) Parodi \\
\hline & & Nassella mucronata (Kunth) R.W. Pohl \\
\hline & & Nassella pubiflora (Trin. \&Rupr.) E. Desv. \\
\hline & & Ortachne erectifolia (Swallen) Clayton \\
\hline
\end{tabular}


día y frígido en la noche, con una temperatura promedio que varía entre los $12^{\circ} \mathrm{C}$ y $15^{\circ} \mathrm{C}$ (Gutiérrez, 2009).

\section{Metodología}

Las colectas de las especies de Poaceae se realizaron siguiendo las técnicas convencionales de colectas botánicas (Cerrate, 1964) y los trabajos de La Torre (1998) y La Torre et al. (2003, 2004). No se realizaron colectas en áreas donde la vegetación presentaba un crecimiento alterado por actividades antrópicas como caminos, terrenos de cultivo abandonados o zonas con evidencia de construcciones. Asimismo, se excluyeron las especies cultivadas.

El periodo de las colectas se realizó entre marzo de 2008 y octubre de 2013. La determinación taxonómica de las muestras se realizó empleando claves y descripciones publicadas en trabajos de la especialidad, como Burkart (1969), Tovar (1957, 1960, 1965, 1972, 1993), Rivas-Martínez \& Tovar (1982) y Rivas-Martínez et al. (1988). El tratamiento nomenclatural se basó en los trabajos de Peterson et al. (2001), Zuloaga et al. (2003), Soreng et al. (2003) y Refulio-Rodríguez et al. (2012). Se ha utilizado la nomenclatura actualizada y las combinaciones nuevas a la luz de los trabajos del Grass Phylogeny Working Group (GPWG, 2001) y revisiones en publicaciones recientes (últimos 5 años).

\section{Resultados.}

Se registró un total de 46 especies y una subespecie de la familia Poaceae agrupadas en 21 géneros, 11 tribus y 6 subfamilias (Tabla 1). Las subfamilias con mayor número de especies son Pooideae con 31 especies y una subespecie, Chloridoideae con 7 especies, Panicoidae con 4 y Danthoniodeae con 2 especies.

En cuanto a la riqueza de especies, el género Calamagrostis es el más diverso con 9 especies, seguido por Poa con 5 especies, mientras que los géneros con menor número de especies son Aristida, Arundo, Avena, Bouteloua, Dactylis, Jarava, Orthacne y Sporobolusy, todos con sólo una especie. De las 46 especies y subespecie que son reportadas, 4 constituyen nuevos registros para la región de Huancavelica (Tovar, 1957, 1993), siendo estas: Aciachne acicularis, Ortachne erectifolia, Arundodonax y Cortaderia hieronymi.

Géneros y especies de Gramíneas (Poaceae) en el distrito de Lircay

1. Raquilla articulada por debajo de las glumas, que caen junto con los granos.

2. Espiguillas siempre unifloras, solitarias sobre el raquis, caen con el pedicelo. Polypogon

2'. Espiguillas bifloras o multifloras, si es uniflora entonces sin las características arriba mencionadas.
3. Espiguillas rodeadas por una o varias cerdas libres o soldadas en la base formando un involucro. Lema fértil no endurecido, no cartilaginoso. Cenchrus

3'. Espiguillas sin cerdas involucrales en su base inflorescencia en espiga o racimo espiciforme unilateral, estos digitados o esparcidos a lo largo del raquis. Lema fértil endurecida, fuertemente cartilaginosa. Paspalum

1'. Raquilla articulada por encima de las glumas, que son persistentes después de la caída de los granos.

4. Espiguilla uniflora.

5. Lema con arista simple.

6. Glumas más largas que la lema, agudas múticas, lema con arista apical.

7. Lema endurecida rígida o papirácea con antopodio pubescente agudo $\mathrm{y}$ punzante.

8. Lema fusiforme, terete con arista algo vilosa en la parte basal y plumosa hacia la parte media $\mathrm{y}$ superior o si no entonces el ápice de la lema con anillo de pelos semirrígidos más largos que la lema y glumas membranáceas. Jarava

8'. Lema fusiforme u obovada, sin la combinación de caracteres anteriores. Nassella

7'. Lema membranácea sin arista o con arista dorsal o subapical, antopodio no pungente. Calamagrostis

6'. Glumas más cortas que la lema, rara vez mayores, múticas o aristadas, lema con arista apical o subapical.

9' láminas rígidas, con ápices punzantes

10. Glumas obtusas, arista de la lema pungente, planta pulviniforme. Aciachne

10'. Glumas truncadas, arista de la lema no pungente, planta cespitosa. Orthacne

9' láminas no rígidas, los ápices no punzantes.

11. Lemas agudas comúnmente aristadas. Muhlenbergia

11'. Lemas obtusas múticas. Sporobolus

5'. Lema con arista trífida en el ápice. Aristida

4'. Espiguillas bi a multifloras.

12. Plantas herbáceas comúnmente menores de 1 metro de altura, espiguillas con lema y raquila glabra o escasamente pubescente.

13. Lemas mucronadas o aristadas.

14. Lema no mucronadas. Avena

14'. Lema mucronada con arista apical o subapical.

15. Glumas desiguales, lema con 
arista apical o subapical mucronada; bilobado o bidentada.

16. Panoja constituida por espiguillas reunidas en densos glomérulos. Dactylis

16'. Panoja constituida por espiguillas que no forma glomérulos.

17. Lema mútica raramente aristada, 5-7 nervadas.

\section{Festuca}

17'. Lema mucronada o aristada, 8-11 nervadas. Bromus

15'. Glumas muy desiguales, la inferior a veces muy pequeña, lema en el ápice con arista larga y recta. Vulpia

13'. Lemas múticas o cortamente aristadas.

18. Inflorescencia en racimos. Bouteloua

18'. Inflorescencia en panícula, contraída o abierta.

19. Paleas persistentes en la raquilla después de caer las glumas y lemas. Eragrostis

19'. Paleas no persistentes en la raquilla después de caer las glumas y lemas. Poa

12'. Plantas leñosas o subleñosas mayores de 1 metro de altura, espiguillas con lema y raquila cubierta por largos pelos sedosos.

20. Espiguillas con flores hermafroditas. Arundo

20'. Espiguillas con flores unisexuales (dioicas). Cortaderia

Para cada especie se incluyen datos sobre su hábitat, distribución, material estudiado y nombre común cuando se conozca; los nombres comunes en idioma quechua han sido escritos tal como suenan.

\section{Aristida L.}

Género agrupado por cerca de 250 especies de zonas tropicales y subtropicales. En el Perú se encuentran registradas 12 especies. Para el área estudiada solo una especie.

\section{Aristida adscensionis $\mathrm{L}$.}

En la zona de estudio se encuentra entre los 32003240 m, en áreas asociadas a Baccharis sp. y Agave cordillerensis. Material estudiado: H. Gutiérrez 0089

(3200 m).

Arundo L.

Género conformado por 3 especies de la región mediterránea hasta el Oriente. Introducida en América En el Perú se registra una sola especie naturalizada.

\section{Arundo donax L.}

En la zona de estudio se encuentra entre los 3200$3260 \mathrm{~m}$, en áreas de permanente humedad, cercano a campos de cultivo y ocasionalmente en riberas de los ríos. Material estudiado: H. Gutiérrez 0020 (3260 m). Nombre común: Carrizo.

\section{Bouteloua Lag.}

Género con aproximadamente 39 especies en el mundo. Es reconocido como uno de los géneros más importantes de la zona central y sureste de Norteamérica presente en las formaciones de praderas y planicies con gramíneas. En el Perú cinco especies.

3. Bouteloua simplex Lag.

En Lircay es frecuente en lugares moderadamente secos por debajo de los $3400 \mathrm{~m}$. Material estudiado: H. Gutiérrez 0104 (3000-3600 m).

\section{Eragrostis Wolf.}

Género con más de 350 especies de los trópicos y sub trópicos del mundo. En el Perú se conocen 29 especies. En la zona de estudio se registraron2 especies, una determinada y la otra en proceso.

4. Eragrostis nigricans (Kunth) Steud.

En la zona de estudio se encuentra entre los 3200 $3600 \mathrm{~m}$, en áreas alteradas, bordes de camino. Material estudiado: H. Gutiérrez 0034 (Constancia, 3780 m). Nombre común: No reportado.

5. Eragrostis sp.

En la zona de estudio se encuentra entre los 3200$3600 \mathrm{~m}$, en áreas alteradas, bordes de camino. Material estudiado: H. Gutiérrez 0035 (3300 m).

\section{Muhlenbergia Schreb.}

Género con más de 160 especies, la mayoría americanas. En el Perú se registran 13 especies y para la zona tres especies.

1. Hierba perenne, cespitosa, lemas múticas, glumas enteras.

2. Hierba fuertemente rizomatosa, rastrera; láminas foliares involutas; glumas agudas.

Muhlenbergia fastigiata

2'. Hierba no rizomatosa, decumbente; láminas foliares planas o sub involutas; glumas obtusas. Muhlenbergia ligularis

1'. Hierba anual, lemas aristadas, arista flexuosa, gluma superior 3-dentada.

Muhlenbergia peruviana 


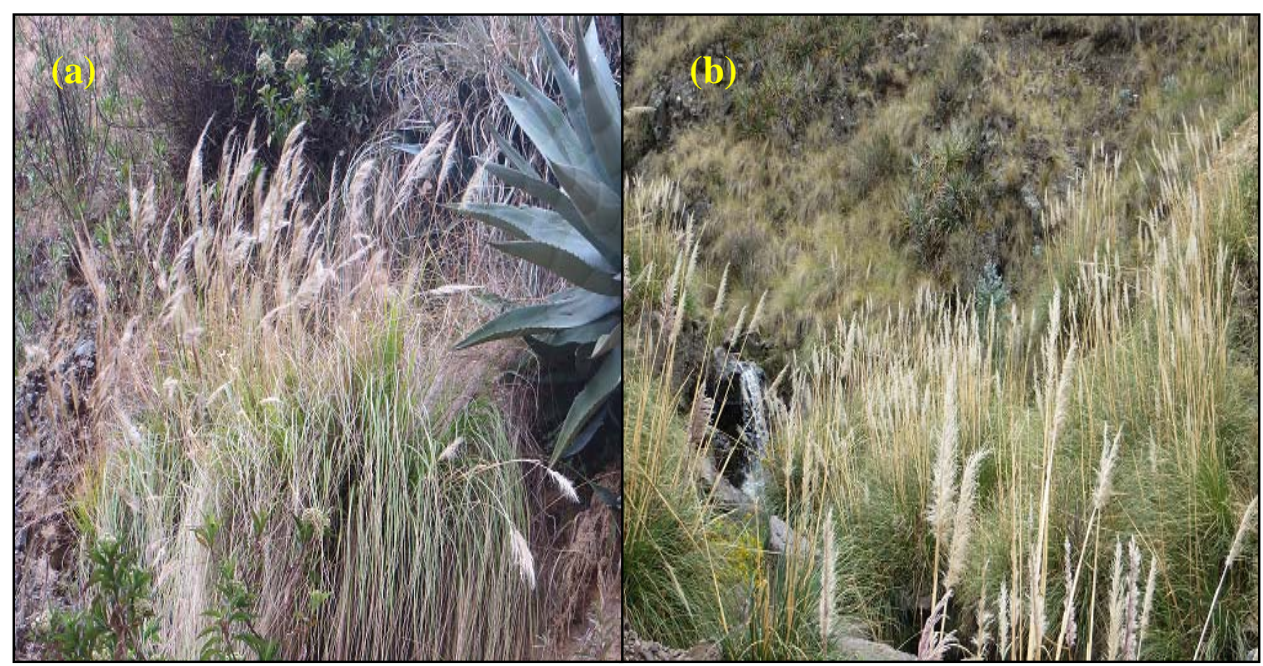

Figura 2. (a) Cortaderia hieronymi N.P. Barker \& H. P. Linder, sector Llajuapampa. (b) Cortaderia jubata (Lemoine) Stapf, sector carretera a Carhuapata a $4.8 \mathrm{~km}$ de la capital del distrito de Lircay. (Fotografías R. Castañeda 2013).

\section{Muhlenbergia fastigiata (J. Presl) Henrard}

En la zona de estudio se encuentra entre los 3800 $4200 \mathrm{~m}$, en áreas de pastoreo. Material estudiado: $\mathrm{H}$. Gutiérrez 0037 (Constancia, $3880 \mathrm{~m}$ ).

\section{Muhlenbergia ligularis (Hack.)} Hitchc.

En la zona de estudio se encuentra entre los $3400-$ $4200 \mathrm{~m}$, en áreas de pastoreo y suelos degradados. Material estudiado: H. Gutiérrez 0045 (Constancia, $3880 \mathrm{~m})$.

\section{Muhlenbergia peruviana (P. Beauv.)} Steud.

En la zona de estudio se encuentra entre los $3750-$ $4200 \mathrm{~m}$, en áreas de pastoreo. Material estudiado: $\mathrm{H}$. Gutiérrez 0018 (Constancia, $3880 \mathrm{~m}$ ).

\section{Sporobolus R.Br}

Género con cerca de 100 especies de los trópicos y sub trópicos del mundo. En el Perú se han registrado 15 especies, en la zona de estudio una.

\section{Sporobolus indicus (L.) R.Br.}

Especie muy común en los campos abiertos y bordes de camino entre los 3200-3600 m. En las zonas es común encontrarlo asociado al pasto "kikuyo" (Cenchrus clandestinus). Material estudiado: $\mathrm{H}$. Gutiérrez 0043 (3260 m).

\section{Cortaderia Stapf}

Género conformado por 26 especies, mayormente de Sudamérica, con algunas especies en Nueva Zelanda y Nueva Guinea. En el área de estudio se registraron dos especies (Figura 2).
1. Lema no dentada, no aristada, glumas de $10-12 \mathrm{~mm}$.

Cortaderia jubata

1'. Lema profundamente dentada, aristada, gluma menores de $10 \mathrm{~mm}$, frecuentemente de $4 \mathrm{~mm}$.

Cortaderia hieronymi

10. Cortaderia hieronymi N.P. Barker \& H.P. Linder

En la zona de estudio se encuentra entre los 3200$3400 \mathrm{~m}$, en áreas rocosas y con presencia de constantes procesos erosivos. El ejemplar colectado presenta el tamaño de las glumas menores a $4 \mathrm{~mm}$, las aristas laterales son más grandes $(25 \mathrm{~mm})$ que en la descripción del ejemplar tipo. Material estudiado: $\mathrm{H}$. Gutiérrez 0101 (3240 m).

11. Cortaderia jubata (Lemoine) Stapf

En la zona de estudio se encuentra entre los 3200$3800 \mathrm{~m}$, en áreas de permanente humedad, bordes de río y manantiales, es una especie colonizadora de áreas de disturbios erosivos. Material estudiado: $\mathrm{H}$. Gutiérrez 0026 (Constancia, 3780 m). Nombre común: jesje.

\section{Cenchrus L.}

Históricamente los géneros Pennisetum Rich. Y Cenchrus L., fueron tratados en forma separada, sin embargo estudios filogenéticos de la tribu Paniceae mostraron que ambos géneros forman un clado monofilético de alto soporte (Gutiérrez \& Morrone, 2012) donde todas las especies de Cenchrus resultaron anidadas dentro del clado que incluía a Pennisetum. Por ende muchas de las especies antes tratadas dentro 
de Pennisetum fueron transferidas a Cenchrus (Chemisquy et al., 2010).

1. Planta estolonífera, rastrera, espigas poco visibles incluidas en las vainas superiores, hojas lineales.

Cenchrus clandestinus

1'. Planta cespitosa, erguida, espigas excertas, hojas sagitadas.

Cenchrus weberbaueri

12. Cenchrus clandestinus (Hochst. ex Chiov.) Morrone.

Especie naturalizada, de amplia distribución entre los 3200-4000 m, en la zona es común encontrarlo asociado a campos abandonados y como especie invasora en pastizales con buen drenaje y régimen hídrico. Material estudiado: H. Gutiérrez 0107 (3260 m). Nombre común: Kikuyo.

13. Cenchrus weberbaueri (Mez) Morrone

Especie distribuida entre los 3200-3600 m, en la zona es común encontrarlo asociado a Agave cordillerensis, se presenta como una especie ruderal, en Lircay únicamente se encuentra en la zona denominada de "ucu-ato". Material estudiado: H. Gutiérrez 0100 (3240 m). Nombre común: Tuntuy, sarasara.

\section{Paspalum L.}

Género con aproximadamente 330 especies. En el Perú se reportan 49 especies y en la zona de estudio dos.

1. rizomatosa-tuberosa, raquis de los racimos conspicuamente membranáceo o foliáceo, alado; el ancho del raquis mayor que el largo de la espiguilla.

Paspalum tuberosum

1'. no rizomatosa-tuberosa, raquis de los racimos no conspicuamente membranáceo, foliáceo $\mathrm{o}$ alado, el ancho del raquis no mayor que el largo de la espiguilla.

Paspalum pilgerianum

\section{Paspalum pilgerianum Chase}

En la zona de estudio se encuentra entre los 3300$3600 \mathrm{~m}$, en áreas de pastoreo. Material estudiado: H. Gutiérrez 0033 (3700 m). Nombre común: Sara-sara.

\section{Paspalum tuberosum Mez}

En la zona de estudio se encuentra entre los 3600$3700 \mathrm{~m}$, en áreas de pastoreo. Material estudiado: H. Gutiérrez 0028 (3650 m).

\section{Calamagrostis Adans.}

Género con aproximadamente 270 especies, en regiones templadas y también en las montañas de los trópicos. En el Perú se registran 43 especies, en la zona de estudio es el género con mayor riqueza ( 8 especies).
1. Raquilla prolongada entre las glumas y el antecio.

2.Panículas oblongas, hojas involutas, rígidas, glumas de 5-7.5 mm.

Calamagrostis chrysantha

2'. Panículas aovadas, hojas subinvolutas, suaves, glumas de 7.8-13 mm.

Calamagrostis ovata

1'. Raquilla no prolongada entre las glumas y el antecio.

3. Raquilla con pelos densos tan largos o iguales como el largo de la lema.

4. Panículas menores de $8 \mathrm{~cm}$, pedicelos pubescentes o pilosos.

Calamagrostis jamesonii

4'. Panículas mayores de $8 \mathrm{~cm}$, pedicelos no pubescentes.

5. Panícula densa, ramas adpresas, láminas fuertemente engrosadas de $1 \mathrm{~mm}$ de grosor.

Calamagrostis rigida

5'. Panícula laxa, ramas extendidas, laminas angostas menores de 0.8 $\mathrm{mm}$.

Calamagrostis intermedia

3'. Raquilla con pelos ralos o glabra, de menor tamaño que la lema.

6. Raquilla glabra, menor a $1 \mathrm{~mm}$, cañas de $10-30 \mathrm{~cm}$.

Calamagrostis rigescens

$6^{\prime}$. Raquilla pubescente, mayor a $1 \mathrm{~mm}$

7. Cañas menores de $5 \mathrm{~cm}$, láminas de 0,5-1 cm de largo.

Calamagrostis minima

7'. Cañas mayores de $5 \mathrm{~cm}$, láminas mayores a $2 \mathrm{~cm}$ de largo.

8. Ramas de las panícula floridas desde la base, laminas involutas no escabrosas, algunas veces filiformes, arqueadas, lema de $3.5 \mathrm{~mm}$ de largo, raquilla de $0,5 \mathrm{~mm}$.

Calamagrostis vicunarum

8'. Ramas de las panículas desnudas en la base, láminas involutas escabrosas, rígidas, lemas de $4.8 \quad-5.4 \mathrm{~mm}$ de largo, raquilla de $2-3 \mathrm{~mm}$.

Calamagrostis recta

16. Calamagrostis chrysantha (J. Presl) Steud.

Es una especie que crece por encima de los 4000 $\mathrm{m}$ en áreas de bajo o nulo drenaje, zonas pantanosas, semipantanosas o las orillas de lagos altos andinos. Es utilizada como forraje en estadios tiernos; forma densos manojos característicos. Material estudiado: $\mathrm{H}$. 
Gutiérrez 0036 (Carhuapata, 4100 m). Nombre común: Ichu.

17. Calamagrostis intermedia (J. Presl) Steud.

Es una de las especies de mayor dominancia en los pajonales, forma densos manojos. Es utilizada para el techado de las casas y "chozas" y como forraje en estadios tiernos (brotes tiernos después de las quemas). Material estudiado: H. Gutiérrez 0035 (3600 m). Nombre común: Ichu.

18. Calamagrostis jamesonii Steud.

Es una especie presente en las zonas de pajonal de puna asociada a suelos húmedos, bordes de turberas y bofedales de puna. H. Gutiérrez 0132 (Cerca de Licapa, $4300 \mathrm{~m}$ ).

19. Calamagrostis minima (Pilg.) Tovar.

Especie presente en bordes de cursos de agua, constituye un buen forraje para el ganado auquénido, forma matas densas de $5-10 \mathrm{~cm}$ de alto. Material estudiado: H. Gutiérrez 0125 (Carhuapata, $4400 \mathrm{~m}$ ). Nombre común: Champa.

20. Calamagrostis recta (Kunth) Trin. ex Steud.

Es una especie afín a Calamagrostis intermedia, ambas constituyen los elementos florísticos más importantes en los pajonales de puna. Algunas veces es usada para forraje únicamente a partir de brotes tiernos. Material estudiado: H. Gutiérrez 0130 (Carhuapata, 4100 m). Nombre común: Huayllaichu.

21. Calamagrostis rigescens (J. Presl) Scribn.

Es una especie afín a Calamagrostis vicunarum, ambas constituyen los elementos florísticos importantes en las zonas de bofedales de puna, o bordes de cursos de agua. Material estudiado: $\mathrm{H}$. Gutiérrez 0109 (Carhuapata, 4100 m).

22. Calamagrostis rigida (Kunth) Trin. ex Steud.

Es una especie con amplia distribución en los pajonales de puna, frecuentemente presente con especies pulviniformes, campos abiertos. Material estudiado: H. Gutiérrez 0122 (Carhuapata, 4200 m). Nombre común: Ichu.

23. Calamagrostis ovata (J. Presl) Steud.

Es una especie presente en las partes húmedas, bordes de manantiales, lagunas de la puna alta. Material estudiado: H. Gutiérrez 0134 (Cerca de Licapa, 4300 m). Nombre común: Sora huayta.

24. Calamagrostis vicunarum (Wedd.) Pilg.

Es una especie abundante en las zonas de bofedales de puna, o bordes de cursos de agua. Constituye un buen forraje para el ganado auquénido. Material estudiado: H. Gutiérrez 0125 (Carhuapata, 4100 m). Nombre común: Crespillo, cushpa-cushpa.

\section{Bromus L.}

Género con aproximadamente 150 especies distribuidas en regiones templadas de ambos hemisferios. En el Perú se registran 8 especies. En la zona de estudio se han registrado dos especies.

1. Espiguillas conspicuamente comprimidas; lemas glabras, escabrosas o esparcidamente pubescentes en el nervio central, aristada, arista menor de $5 \mathrm{~mm}$ de largo.

Bromus catharticus

1'. Espiguillas no conspicuamente comprimidas; lemas pubescentes, arista menor de $5 \mathrm{~mm}$ de largo o sin arista.

Bromus pitensis

\section{Bromus catharticus Vahl}

Esta especie crece en los bordes de terrenos de cultivos y pastizales, campos abiertos; la altitud mayor registrada es a los $4000 \mathrm{~m}$. Material estudiado: $\mathrm{H}$. Gutiérrez 0032 (3200 m). Nombre común: Cebadilla, socclla.

\section{Bromus pitensis Kunth}

Esta especie está asociada a lugares sombreados, entre arbustos. Al parecer solo de los Andes del Ecuador, Perú y Bolivia. Se encuentra en los niveles medios entre los 3200-3800 m. Material estudiado: H. Gutiérrez 0096 (3260 m). Nombre común: Socclla.

\section{Avena L.}

Género con 25 especies distribuidas en el Mediterráneo y Medio Oriente, existiendo al norte de Europa. Introducida en otras regiones. En el Perú se consideran cuatro especies, y en la zona de estudio una.

\section{Avena sterilis L.}

En la zona de estudio se encuentra entre los 3600 $3700 \mathrm{~m}$, en áreas cercanas a campos de cultivo abandonados, suelos con mediana humedad. Material estudiado: H. Gutiérrez 0057 (3600 m). Nombre común: Cebadilla.

\section{Dactylis L.}

Género con una sola especie nativa de la zona templada de Eurasia, introducida en América, principalmente con fines de forraje.

28. Dactylis glomerata L.

Especie que se presenta en bordes de camino aledaño a zonas de pastoreo entre los 3200-3600. Material estudiado: H. Gutiérrez 0127 (3600 m). Nombre común: Pasto dactilis, pasto inglés.

\section{Festuca L.}

Género con aproximadamente 450 especies, de regiones templadas extendiéndose a las montañas de los trópicos. En el Perú se registran 42 especies y para la zona de estudio dos. 
1. Planta perenne, amacollada, con cañas de más de $50 \mathrm{~cm}$ de altura, glumas no atenuadas o ligeramente acuminadas; espiguillas generalmente de 9-11 mm de largo.

Festuca dolichophylla

1'. Planta perenne, en manojos, con cañas de 20$50 \mathrm{~cm}$ de altura, glumas atenuadas hacia el ápice; espiguillas generalmente de 7-8.5 mm de largo.

Festuca weberbaueri

29. Festuca dolichophylla J. Pres1

Especie densamente amacollada, muy frecuente en la formación de pajonal, forma comunidades de gramíneas amacolladas, ampliamente distribuida entre los 3800-4500 m. Material estudiado: H. Gutiérrez 0137 (Carhuapata, $3890 \mathrm{~m}$ ). Nombre común: Chillhua, ichu.

30. Festuca weberbaueri Pilg.

Especie muy frecuente en la formación de pajonal con arbustos dispersos, forma comunidades junto a especies arbustivas en laderas pedregosas, ampliamente distribuida entre los 3700-4200 m. Material estudiado: H. Gutiérrez 0139 (Constancia, $3700 \mathrm{~m})$.

Poa L.

Género con 500 especies, propias de las regiones templadas y frías de ambos hemisferios. En el Perú se reportan 41 especies (Poa s.s.) y en la zona de estudio seis.

1. Planta anual

2. Lema pubescente (en la mitad inferior), de 2-4.5 mm. Glumas desiguales, la inferior de 1,5- $2 \mathrm{~mm}$ y la superior de 2-2,5 $\mathrm{mm}$ de largo.

Poa annua

2'. Lema glabra, de 2,2-2,5 mm. Glumas iguales, de 2,8-3,5 $\mathrm{mm}$ de largo.

Poa serpaiana

1'. Planta perenne

3.Lema flabelada

3'. Lema no flabelada

Poa lepidula

4. Planta robusta, con cañas de $30-90 \mathrm{~cm}$ de altura,

Poa horridula

4'. Planta no robusta, con cañas de 2,5 - 8 $\mathrm{cm}$ de altura.

Poa calycina

\section{Poa annua L.}

Introducida de Europa, ampliamente difundida en el Perú, desde la costa hasta la puna inferior, en lugares sombreados y bordes de camino, se la puede encontrar por encima de los $3900 \mathrm{~m}$. Material estudiado: H. Gutiérrez 0012 (3200 m). Nombre común: Kcehua.
32. Poa calycina(J. Presl) Kunth

Especie tratada anteriormente como Dissanthelium calycinum (J. Presl) Hitchc. Perenne, con cañas de 2,5-8 cm de altura, frecuente en los pajonales de Puna, suelos algo secos entre los 3900-4500 m. Material estudiado: H. Gutiérrez 0149 (Carhuapata, 3950 m).

33. Poa horridula Pilg.

Especie asociada a suelos rocosos, habita entre los 3000-3850 m. Material estudiado: H. Gutiérrez 0153 (C.C. Cóndor Paccha, 4000 m).

34. Poa lepidula (Nees \& Meyen) Soreng \& L.J. Gillespie

La especie se encuentra por encima de los $4500 \mathrm{~m}$, en áreas rocosas, suelos crioturbados. Material estudiado: H. Gutiérrez 0004 corresponde a un ejemplar de la zona carretera a Julcani. (Lircay, C.P. Julcani) $4540 \mathrm{~m}$. Especie anteriormente tratada bajo un género monotípico (Anthochloa Nees \& Meyen).

35. Poa serpaiana Refulio

Especie tratada anteriormente como Dissanthelium peruvianum (Nees \& Meyen) Pilg. Anual, con cañas algo geniculadas en la base, de $2.5-13 \mathrm{~cm}$ de altura, presente en los pajonales de puna con arbustos muy dispersos, suelos húmicos entre los $3750-4200 \mathrm{~m}$. Material estudiado: H. Gutiérrez 0160 (Carhuapata, $3900 \mathrm{~m})$.

\section{Polypogon Desf.}

Género con 18 especies, en el Perú se registran 5 especies y en la zona de estudio dos.

1. Glumas gradualmente angostadas hacia el ápice que terminan en una arista corta, de $1.5-2.5 \mathrm{~mm}$

Polypogon elongatus

1'. Glumas redondeadas en el ápice que terminan en una arista larga, mayor de $2,5 \mathrm{~mm}$

Polypogon interruptus

36. Polypogon elongatus Kunth

Esta especie en la zona de estudio se encuentra entre los 3200-3700 m, en áreas cercanas a campos de cultivo abandonados, suelos con alta humedad. Material estudiado: H. Gutiérrez 0057 (3600 m).

37. Polypogon interruptus Kunth

En la zona de estudio se encuentra entre los 3200$3700 \mathrm{~m}$, en áreas cercanas a zonas anegadas. Material estudiado: H. Gutiérrez 0017 (3600 m).

\section{Aciachne Benth}

Género distribuido en los altos Andes con tres especies desde Venezuela hasta Bolivia y Argentina. En el Perú se reportan dos especies.

1. Espiguillas de 5.5 a $6 \mathrm{~mm}$ de largo, glumas de 3.0 a $3.5 \mathrm{~mm}$, cespitosas, no estoloníferas

Aciachne pulvinata 
1'. Espiguillas de 4.0 a $4.5 \mathrm{~mm}$ de largo, glumas de 1,5 a $2,5 \mathrm{~mm}$, cespitosas o estoloníferas.

Aciachne acicularis

38. Aciachne acicularis Lægaard

Especie pulviniforme muy afín a A pulvinata de la cual difiere por las glumas de menor tamaño. Forma cojines en zonas de pastoreo frecuente y en pajonales de baja palatabilidad, en suelos con baja humedad. Material estudiado: H. Gutiérrez 0014 (Constancia, 3800 m). Nombre común: Paccupaccu.

39. Aciachne pulvinata Benth

Especie pulviniforme asociada a hábitats deteriorados por sobre pastoreo, forman cojines densos de forma circular conformando almohadillados en zonas húmedas y turberas. Material estudiado: $\mathrm{H}$. Gutiérrez 0008 (Constancia, 3760 m). Nombre común: Paccupaccu.

\section{Jarava Ruiz \& Pav.}

Género con alrededor de 300 especies de zonas templadas y templado-cálidas del mundo, anteriormente tratadas como Stipa. En el Perú se registran 13 especies y una en la zona de estudio.

40. Jarava ichu Ruiz \& Pav.

Parte altoandina, formando grandes pajonales $\mathrm{o}$ comunidades, siendo el elemento típico de la puna en el caso del Perú, es la especie más difundida de los Andes peruanos. Material estudiado: H. Gutiérrez 0170 (3260 m). Nombre común: Ichu, peccoy.

\section{Nassella (Trin) Desv.}

Se registran 109 especies a nivel mundial. Varias especies han sido segregadas del género Stipa, todas nativas del nuevo mundo. En el Perú se registran aproximadamente 16 especies y en la zona de estudio tres

1. Lema obovada, oblongo o cuneiformecilindráceo, menor de $2 \mathrm{~mm}$ de largo, arista menor a $30 \mathrm{~mm}$.

2.Lema obovada de 1,5-2 $\mathrm{mm}$ de largo, arista geniculada de $15 \mathrm{~mm}$.

Nasella pubiflora

2'. Lema oblonga o cuneiforme-cilindráceo de 1,7-2 $\mathrm{mm}$ de largo, la arista de 10-15 $\mathrm{mm}$.

Nassella meyeniana

1 '. Lema terete o fusiforme de 4,5-9 $\mathrm{mm}$ de largo, arista bigeniculada de $30-45 \mathrm{~mm}$.

Nasella mucronata

\section{Nasella meyeniana (Trin. \&Rupr.)} Parodi

Especie distribuida entre los 3100-3800 m en los flancos occidentales de los andes y los valles interandinos. Se caracteriza por tener una arista muy caediza, frecuente en bordes de camino y zonas de mediana humedad. Material estudiado: H. Gutiérrez 0034 (3260 m).

42. Nasella mucronata (Kunth) R.W. Pohl

Especie anteriormente tratada como Stipa mucronata Kunth. Distribuida entre los 3100-3800 m en los flancos occidentales de los Andes y los valles interandinos; frecuente en bordes de camino y zonas de mediana humedad. Material estudiado: $\mathrm{H}$. Gutiérrez 0016 (3260 m).

43. Nasella pubiflora (Trin. \& Rupr.) E. Desv.

Especie ampliamente distribuida, en bordes de camino y en campos de cultivos abandonados. En la zona es frecuente entre los 3200-3400 m comúnmente en zonas de moderada pendiente. Material estudiado: H. Gutiérrez 0124 (3260 m).

\section{Ortachne Nees ex Steud}

Género constituido por ocho especies distribuidas desde Costa Rica hasta la Argentina. En Chile se registran dos especies nativas, en el Perú una sola especie, anteriormente tratada dentro del género monotípico Lorenzochloa Reeder \& C. Reeder.

\section{Ortachne erectifolia (Swallen) Clayton}

Es el componente florístico más importante de los pajonales altoandinos de la provincia. Especie usada como techo para casas rurales y en la confección de escobas "pichanas". Material estudiado: H. Gutiérrez 0002 (3400 m).Nombre común: Iruichu, ichu fuego.

\section{Vulpia C. C. Gmel.}

Género con cerca de 22 especies, propias de regiones templadas, y subtropicales de los hemisferios Norte y Sur. En el Perú se registran cuatro especies y en la zona de estudio tres.

1. Gluma inferior igual o más larga que la mitad de la gluma superior.

Vulpia australis

1'.Gluma inferior igual al tercio de la gluma superior o más pequeña.

2. Lema con los bordes pilosos en la mitad superior.

Vulpia myuros

$2^{\prime}$. Lema con los bordes totalmente glabros Vulpia myuros var. myuros

45. Vulpia australis (Nees ex Steud.) C.H. Blom

Esta especie está asociada a partes sombreadas, en terrenos pedregosos de preferencia. En Lircay se encuentra en los niveles medios. Difiere de V. myuros por encontrarse en zonas con menor humedad. Material estudiado: H. Gutiérrez 0164 (Carhuapata, $3980 \mathrm{~m})$. 


\section{Vulpia myuros Hack}

Variedad restringida a suelos algo sombreados, borde de cultivos y camino. Distribuida del oeste de Estados Unidos de Norteamérica hasta la Argentina. En Lircay ampliamente distribuida, ocupando los niveles medios entre $3200-3600 \mathrm{~m}$.

Material estudiado: H. Gutiérrez 0016 (3400 m).

47. Vulpia myuros (L.) C.C. Gmel var. myuros

Esta variedad habita en suelos húmedos, algo sombreados, entre arbustos. Es de origen europeo, naturalizada en la América tropical, de clima templado. En Lircay está difundida en la parte media de la cuenca del Sicra y del Opamayo en bordes de camino. Material estudiado: H. Gutiérrez 0004 (3400 $\mathrm{m})$.

\section{Discusión.}

Tovar en 1957 registra 88 especies de Poaceae para la región Huancavelica. En el presente estudio se reportan 46 especies y una variedad de Poaceae para Lircay, cuatro de las cuales constituyen nuevos registros para la región. A nivel distrital y provincial es el primer trabajo en su tipo, puesto que anteriormente no se consignaron datos sobre la riqueza agrostológica en la provincia de Angaraes. En tal sentido, con el presente estudio se incrementa el registro de especies a nivel regional. Además, se han consignado los cambios nomenclaturales; estas segregaciones y combinaciones nuevas se deben a que este estudio tiene un paralelo de más de 50 años con los trabajos de Tovar (1957), quien realizó sus colectas en las provincias de Huancavelica y Tayacaja, sin colectas en Angaraes (Lircay). El hecho de haber colectado las muestras en áreas poco o no perturbadas indica que su distribución es natural; sin embargo, es preciso mencionar que hay especies que han sido naturalizadas ampliamente, constituyendo elementos florísticos comunes como son Poa annua, Cenchrus clandestinus y Arundodonax (en menor frecuencia). Finalmente, ninguna de las 13 especies de gramíneas consideradas endémicas en la región (La Torre et al, 2006) han sido registradas en el área de estudio.

\section{Conclusiones.}

Con el presente documento se ha incrementado en cuatro el número de registros de especies de la familia Poaceae para la región Huancavelica (Aciachne acicularis, Arundo donax, Cortaderia hieronymi y Ortachne erectifolia) y se presenta un tratamiento actualizado para los principales taxa de Poaceae.

\section{Agradecimientos.}

Expresamos nuestro agradecimiento y admiración al Dr. Óscar Tovar $(\dagger)$ quien en el año 2008 y primeros meses del 2009 revisó el material colectado.

\section{Literatura citada.}

Brako L. \& Zarucchi J. 1993. Catalogue of the Flowering Plants and Gymnosperms of Peru. Missouri Botanical Gardens, St. Louis, MO.1286 pp.

Burkart A. 1969. Flora ilustrada de Entre Ríos (Argentina). Parte II. GRAMÍNEAS La familia botánica de los pastos. Colección Científica del INTA 6 (2): 1-551.

Cerrate E. 1964. Manera de preparar plantas para un herbario. UNMSM. Museo de Historia Natural, Serie de Divulgación 1.

Chemisquy M.A., Giussani L.M., Scataglini M.A., Kellogg E.A. \& Morrone O. 2010. Phylogenetic studies favour the unification of Pennisetum, Cenchrus and Odontelytrum (Poaceae): A combined nuclear, plastid and morphological analysis, and nomenclatural combinations in Cenchrus. Annals of Botany 106: $107-$ 130.

Clayton W.D. \& Renvoize S.A. 1986. Genera Graminum: Grasses of the World. Kew Bulletin Additional Series 13, Royal Botanical Garden, Kew. Her Majesty's Stationery Office, London. 389 pp.

GPWG (Grass Phylogeny Working Group). 2001. Phylogenyandsubfamilial classification of the grasses (Poaceae). Ann. Missouri Bot. Gard. 88: 373-457.

Gutiérrez H. 2009. Efectos del sobrepastoreo en pastizales de altitud en la Provincia de Angaraes-Departamento de Huancavelica. Tesis para optar el título Profesional de Biólogo. Escuela académico profesional de Biología, Facultad de Ciencias, Universidad Nacional "San Luis Gonzaga de Ica". Ica-Perú. 83 pp.

Gutiérrez H.F. \& Morrone O. 2012. Novedades nomenclaturales en Cenchrus (Poaceae: Panicoideae: Paniceae).Bol. Soc. Argent. Bot. 47 (1-2): 263-269.

INEI. 2010. (en línea). Información de Ubicación Geográfica

$<\mathrm{http}$ ://iinei.inei.gob.pe/iinei/siscodes/UbigeoMarco.htm $>$. Acceso 03/06/2013.

La Torre M.I. 1998. Estudio Taxonómico de la Familia Poaceae del Parque Nacional Yanachaga-Chemillen (Oxapampa, Pasco, Perú).Tesis para optar el grado de Magister en Botánica Tropical Mención Taxonomía y Sistemática. Escuela académico profesional de Ciencias Biológicas, Facultad de Ciencias Biológicas, Universidad Nacional Mayor de San Marcos. LimaPerú.

, Cano A. \& Tovar O. 2003. Las Poáceas del Parque Nacional Yanachaga-Chemillén, Oxapampa, Pasco, Perú. Parte I: Bambusoideae. Rev. peru. biol.10 (2): $145-154$

Cano A. \& Tovar O. 2004. Las Poáceas del Parque Nacional Yanachaga-Chemillén, Oxapampa, Pasco, Perú. Parte II: Pooideae, Centothecoideae, Arundinoideae, Chloridoideae y Panicoide. Rev. peru. biol.1 (1): 51 - 70

, Alegría J. \& Refulio N. 2006. Poaceae endémicas del Perú. En B. León et al (ed). El libro rojo de las plantas endémicas del Perú. Rev. peru. biol. Número especial 13(2): 879 - 891.

Peterson P.M., Soreng R.J., Davidse G., Filgueiras T.S., Zuloaga F.O. \& Judziewicz E. 2001. Catalogue of New World Grasses (Poaceae): II. Subfamily Chloridoideae.Contr.U.S.Nat1.Herb.41:1-255.

Refulio-Rodriguez N.F., Columbus J.T., Guillespie L.J., PetersonP.M. \& Soreng R.J. 2012. Molecular Phylogeny 
of Dissanthelium (Poaceae: Pooideae) and its Taxonomic Implications. Syst.Bot. 37 (1): 122-133.

Rivas-Martínez S. \& Tovar O. 1982. Vegetatio Andinae, 1. Datos sobre las comunidades vegetales altoandinas de los Andes Centrales del Perú. Lazaroa. 4: 167-187. , Tovar O. \& Galán A. 1988. Pisos bioclimáticos y cultivos del Perú 1-69. Informe. Lima.

Soreng R.J., Peterson P.M., Davidse G., Judziewiez E.J., Zuloaga F.O., Filgueiras T.S. \& Morrone O. 2003. Catalogue of New World Grasses (Poaceae): IV. Subfamily Pooideae. Contr. U.S.Natl.Herb. 48:1-730.

Tovar O. 1957. Las gramíneas de Huancavelica, primera parte. Mem. Mus. Hist. Nat. "Javier Prado".6:1-110.

.1960. Revisión de las especies peruanas del género Calamagrostis. Mem. Mus. Hist. Nat. "Javier Prado". 11: 1-91.
.1965. Revisión de las especies peruanas del género Poa. Mem. Mus. Hist. Nat. "Javier Prado". 15:1-67.

.1972. Revisión de las especies peruanas del género Festuca. Mem. Mus. Hist. Nat. "Javier Prado". 16:1-95. .1993. Las Gramíneas (Poáceas) del Perú. Ruizia.13:1-481.

Ulloa C., Zarucchi J. \& León B. 2004. Diez años de adiciones a la Flora del Perú: 1993-2003. Arnaldoa (Edic. Espec.): 1-242.

Zuloaga F.O., Morrone O., Davidse G., Filgueiras T.S., Peterson P.M., Soreng R.J. \& Judziewicz E. 2003. Catalogue of New World Grasses (Poaceae): III. Subfamilies Panicoideae, Aristidoideae, Arundinoideae and Danthonioideae. Contr. U.S. Natl. Herb.46:1-662.

${ }^{1}$ Ministerio del Ambiente. Dirección General de Diversidad Biológica. Av. Javier Prado Oeste 1440, San Isidro. Lima. Apartado Postal 18-0923, Lima 18 - Perú. gutierrezpe5@hotmail.com

${ }^{2}$ Universidad Nacional Agraria la Molina, Facultad de Ciencias, Departamento académico de Biología, Herbario A. Weberbauer (MOL), Av. La Molina s/n. La Molina. Lima. Apartado Postal 12-056, Lima 12 - Perú 